\title{
Prevenga la Osteoporosis: Atrape al Ladrón Silencioso ${ }^{1}$
}

Linda B. Bobroff ${ }^{2}$

\section{¿Porque Preocuparse?}

La osteoporosis ha sido llamada "El ladrón silencioso." Se roba el hueso de las mujeres (y algunos hombres) sin señal Eventualmente, un pequeño

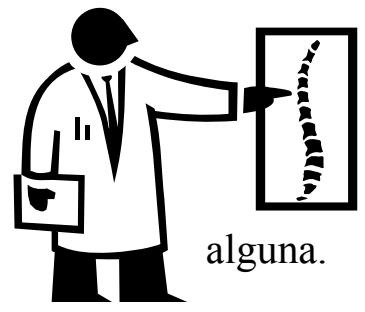
tropezón podría causar una fractura de cadera. Las fracturas espinales pueden resultar en una postura empinada conocida como "joroba."

La osteoporosis son huesos porososHuesos con muchas aberturas (poros) que se pueden partir sin advertencia.

Alrededor del 50\% de las mujeres y el 25\% de los hombres mayores de 50 podrán sufrir fracturas causadas por la osteoporosis durante su tiempo de vida. Muchas de estas fracturas, especialmente en al espina y la cadera, causan dolor, deshabilitad, y perdida de independencia. Alrededor del 24\% de las personas mayores de 50 que tienen fractura en la cadera mueren en un año después de la fractura.

En los Estados Unidos, la osteoporosis causa aproximadamente $1 \frac{1}{2}$ millón de fracturas por año, primeramente en la cadera, la espina, y la muñeca. Los costos médicos de estas fracturas, incluyendo cuidado medico en la casa, están estimadas en mas o menos $\$ 18$ billones por año. A esta figura le debemos adherir los siguientes impactos;
- Pérdida de entrada de dinero

- Pérdida temporal de independencia

- Pérdida de servicios y productividad

- Estrés en los familiares/cuidadores

- Otros costos de salud

De los pacientes con fracturas de cadera, menos del $50 \%$ recuperan sus funciones "normales."

La osteoporosis es similar a la alta presión arterial en que no muestra síntomas sino hasta que le complicación acorre. No hay cura, así que la prevención es esencial para combatir estas enfermedades.

\section{Hueso-Una Bodega de Minerales}

Muchas veces creemos que el hueso es estático e incambiable. Pero éste constante mente rota, formando un

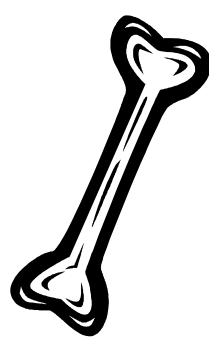
nuevo hueso y descomponiendo el hueso viejo. Cuando la formación y la descomposición del hueso son balanceadas, la cantidad de masa del hueso se mantiene más o menos igual.

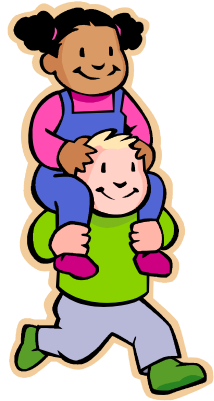

Durante el periodo de crecimiento, hay más formación que descomposición. Desde los treintas en adelante, la descomposición tiende a superar la formación, y hay una lenta y gradual perdida de hueso de ahí en adelante.

1. The English version of this Spanish document is Prevent Osteoporosis: Catch the Silent Thief (FCS8145). Este documento, FCS8145-Span, pertenece a una serie del Departamento de Ciencias de la Familia, Juventud y Comunidad, Servicio de Extensión Cooperativo de la Florida, Instituto de Alimentos y Ciencias Agrícolas, Universidad de la Florida. Fecha de publicación: 1989. Revisado: abril 2007. Favor de visitar el EDIS Web site en el http://edis.ifas.ufl.edu

2. Linda B. Bobroff, Ph.D., RD, LD/N., profesora, Departamento de Ciencias de la Familia, Juventud y Comunidad, Servicio de Extensión Cooperativo de la Florida, Instituto de Alimentos y Ciencias Agrícolas, Universidad de la Florida, Gainesville, FL 32611. 
Ya que la perdida de huesos es un echo, entre mas hueso haya para comenzar, mas hueso van a tener a medida que se van envejeciendo. Usted puede tomar decisiones durante su juventud que le ayudaran a construir masa en los huesos antes de que la pérdida empiece. A medida que usted se va envejeciendo, usted puede disminuir el nivel de descomposición de sus huesos al tomar decisiones positivas para un mejor estilo de vida. A través de nuestra vida, la dieta y el ejercicio son dos cosas que todos podemos controlar para así poder evitar o disminuir el riesgo de osteoporosis.

\section{¿Quien Podría tener Osteoporosis?}

La causa exacta de la osteoporosis es todavía una incógnita. Es conocida como una enfermedad "multifactorial." En otras palabras, hay muchos factores que contribuyen a esta enfermedad. Algunos de estos factores de riesgo de contraer osteoporosis son "innatos" o más allá del control humano. Hay otros factores que se relacionan con el estilo de vida de la persona y estos si pueden ser controlados. Las personas toman decisiones diarias que podrían ayudar a reducir el riesgo de desarrollar osteoporosis.

Los factores de riesgo que no podrían ser controlados son sexo, raza, historia familiar, estatus menstrual, tamaño del cuerpo y ciertas condiciones medicas.

Las mujeres están mas en riesgo de sufrir de osteoporosis que los hombres. Las personas de raza negra corren menos riesgo que los blancos o asiáticos. Aquellas personas con historia familiar de osteoporosis y aquellos de cuerpo pequeño o delgado corren mayor riesgo de sufrir de esta enfermedad. La obesidad parece proteger a las personas de osteoporosis. Esto es causado por la producción de estrógeno en los tejidos de grasa y por el incremento de la carga en el hueso.
Aunque la obesidad proteja contra la osteoporosis, esto incrementa otros riesgos para otras condiciones. Las personas obesas tienden a sufrir de diabetes, problemas cardiovasculares, algunas formas de cáncer y otras condiciones.

En las mujeres, la velocidad en la perdida de hueso incrementa durante la menopausia. Después de más o menos 65 años de edad, esta velocidad disminuye. Las mujeres que paran de menstruar por hacer mucho ejercicio o por problemas de alimentación también pierden hueso.

Varias condiciones médicas incrementan el riesgo de osteoporosis. Personas con problemas de diabetes, problemas de tiroides y aquellos que toman ciertas medicinas que están en alto riego de problema de perdida de hueso. (Vea la Tabla 1.)

Hasta las personas que tienen muchos factores de riesgo podrían disminuir el riesgo de contraer osteoporosis al tomar decisiones de estilo de vida. Estas incluyen tomar bastante calcio, vitamina $\mathrm{D}$, ejercicio para controlar el peso, no fumando, y evadir el exceso de alcohol.

\section{Calcio para Construir Huesos}

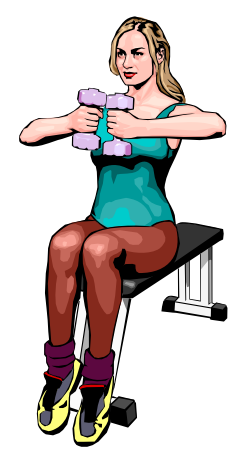

Los huesos se están haciendo y deshaciendo constantemente. Calcio y otros minerales adecuados son esenciales para la reconstrucción del hueso. Por esta razón, es importante consumir una dieta adecuada de calcio, vitamina $\mathrm{D}$, y otros minerales importantes para unos huesos saludables. 


\begin{tabular}{|l|l|}
\hline Tabla 1: Factores de Riesgo de la Osteoporosis \\
\hline Innatos & Relacionados con el estilo de vida \\
\hline Edad mayor & $\begin{array}{l}\text { Muy poquita vitamina D (por la dieta o exposición } \\
\text { solar) }\end{array}$ \\
\hline Mujeres & Muy poco calcio en la dieta \\
\hline Caucáseo o Asiático & Muy poco ejercicio \\
\hline Historia familiar & Uso de Nicotina \\
\hline Menopausia & Uso excesivo de Alcohol \\
\hline Histerectomía & $\begin{array}{l}\text { Medicaciones (esteroides, anti-convulsionantes, } \\
\text { antiácidos con aluminio, extracción de la tiroides) }\end{array}$ \\
\hline Amenorrea (Ausencia del periodo menstrual) & \\
\hline Cuerpo pequeño (menos de 127 libras) & \\
\hline $\begin{array}{l}\text { Desequilibrio químico u hormonal (tales como la } \\
\text { diabetes y problemas de tiroides) }\end{array}$ & \\
\hline \multicolumn{2}{|l}{} \\
\hline
\end{tabular}

Los huesos y los dientes contienen el 99\% del calcio en el cuerpo. El uno por ciento restante es encontrado en la sangre y otros fluidos del cuerpo donde este ayuda la circulación de la sangre y la transmisión de los nervios. El calcio es guardado en los huesos y por causa del bajo consumo, el calcio de los huesos es usado para otras funciones.

La recomendación de consumo diario de calcio es 1,000 miligramos (MG) para una mujer adulta entre los 19 y los 50 y 1,200 para una mujer de 51 o mayor. Jóvenes de 18 para abajo deben consumir 1,300 MG diarios.

\section{Obtener el Calcio de los Alimentos}

La mejor manera de obtener calcio es a través de la dieta. Hay muchos alimentos que son buena fuente de calcio. Incluirlas en las comidas o pasabolas ayudaría a cumplir con el requerimiento de calcio en el cuerpo. La tabla 2 muestra el contenido de calcio de algunos alimentos comunes.
Las comidas lácteas son una excelente fuente de calcio. Para controlar el ingreso de grata, grasa saturada y colesterol, escoja la leche baja en grasa, la leche sin grasa, yogurt o queso.

Otros alimentos tales como los vegetales verdosos, también son una muy Buena fuente de calcio. Escoja el brócoli, los nabos verdes, la col, la col rizada, la mostaza

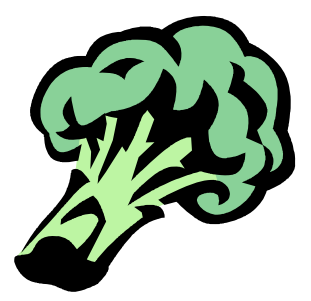
verde, etc. El salmón enlatado (incluyendo los huesos triturados) y las sardinas enlatadas también son una excelente fuente de calcio.

El ácido oxálico en algunos vegetales y el ácido físico y comidas enteras interfieren con la absorción del calcio. Por eso el calcio en los granos enteros, la espinaca, el cardo, la remolacha verde esta menos disponible que el calcio de muchos otros recursos alimenticios. 


\section{Tabla 2. Calcio en Alimentos Seleccionados}

\begin{tabular}{|c|c|c|c|}
\hline Alimento & $\begin{array}{l}\text { Calcio } \\
\text { (mg) }\end{array}$ & Alimento & $\begin{array}{l}\text { Calcio } \\
(\mathrm{mg})\end{array}$ \\
\hline Leche Entera, 1 taza & 290 & Queso parmesano, rallado, 1 onza & 390 \\
\hline Leche baja en grasa, 1\%, 1 taza & 300 & Queso Americano (procesado) 1 onza & 175 \\
\hline Leche sin Grasa, 1 taza & 300 & Queso Coteja Cremoso (1\%), 1 taza & 140 \\
\hline Leche seca sin grasa, 1 cucharada & 60 & Helado (dietético) 1 taza & 180 \\
\hline Leche Chocolateada (1\%) 1, taza & 250 & Jugo de naranja, fortificado, $3 / 4$ taza & $260-450$ \\
\hline Mantequilla de leche (1\%), 1 taza & 285 & $\begin{array}{l}\text { Salmón rosado, enlatado con huesos, } \\
3 \text { onzas }\end{array}$ & 180 \\
\hline Yogurt (bajo en grasa), 1 taza natural & 450 & $\begin{array}{l}\text { Sardinas con huesos (coladas) } 3 \\
\text { onzas }\end{array}$ & 325 \\
\hline $\begin{array}{l}\text { Yogurt (bajo en grasa), } 1 \text { taza con sabor } \\
\text { a frutas }\end{array}$ & 340 & Col (cocinado), 1 taza & 180 \\
\hline Yogurt (bajo en grasa), 1 taza de vainilla & 350 & Brócoli (cocinado), 1 taza & 100 \\
\hline Queso Suizo, 1 onza & 270 & Repollo (Cocinado), 1 taza & 225 \\
\hline Queso Chedar, 1 onza & 200 & Hojas de nabo (cocinado), 1 taza & 250 \\
\hline Queso Mozarela, 1 onza & 180 & Tofu (fortificado), 2 onzas & 280 \\
\hline \multicolumn{4}{|c|}{ Fuente: Food Processor SQL, Version 10.0. ESHA Research, Inc., 2006-07 } \\
\hline
\end{tabular}

La Tabla 3 a continuación provee ejemplos de como incluir alimentos ricos en calcio en nuestra dieta. Las personas que tienen alta necesidad de calcio o que no consumen alimentos lácteos podrían necesitar de ayuda suplemental. 
Tabla 3: Alimentos Ricos en Calcio

\begin{tabular}{|c|c|c|}
\hline Plato & Alimento & $\begin{array}{c}\text { Calcio } \\
\text { (MG) }\end{array}$ \\
\hline
\end{tabular}

DIA 1

\begin{tabular}{|l|l|c|}
\hline Desayuno & $\begin{array}{l}1 \% \text { leche , 3/4 taza de } \\
\text { cereal fortificado, 1 taza }\end{array}$ & 225 \\
\hline Almuerzo & $\begin{array}{l}\text { Lecha Entera, 3 } \\
\text { Cucharadas (Adheridas } \\
\text { al café) }\end{array}$ & 55 \\
\hline Merienda & $\begin{array}{l}\text { Yogurt bajo en grasa } \\
\text { de Vainilla, 1 taza }\end{array}$ & 350 \\
\hline \multirow{2}{*}{ Comida } & $\begin{array}{l}\text { Verduras Cocidas, } 3 / 4 \\
\text { taza }\end{array}$ & 175 \\
\cline { 2 - 3 } & Leche sin grasa, 3/4 taza & 225 \\
\hline \multicolumn{2}{|r|}{ TOTAL } & 1030 \\
\hline
\end{tabular}

DIA 2

\begin{tabular}{|l|l|c|}
\hline Desayuno & $\begin{array}{l}\text { Waffle, fortificado, jugo } \\
\text { de naranja, fortificado, } \\
3 / 4 \text { taza }\end{array}$ & 225 \\
\hline Almuerzo & $\begin{array}{l}\text { Queso Suizo, 1 onza } \\
\text { (En un sándwich) }\end{array}$ & 270 \\
\hline \multirow{2}{*}{ Comida } & Brócoli, 1 taza & 100 \\
\cline { 2 - 3 } & Leche sin grasa, 1 taza & 225 \\
\hline TOTAL & 1080 \\
\hline
\end{tabular}

\section{¿Que tal los Suplementos?}

Los suplementos de calcio están disponibles para aquellos que no consumen suficiente alimentos que contienen calcio. Las dos formas principales de obtener calcio son citrato y carbono. La absorción es similar entre las dos. Sin embargo, el calcio citrato es mejor absorbido por personas con bajo acido estomacal, tales como adultos mayores o personas tomando anti

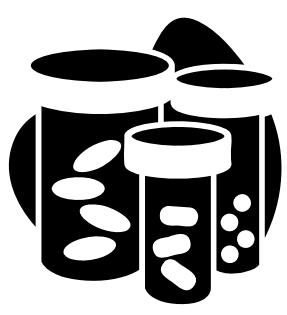
ácidos. Es una buena idea chequear con su doctor o farmaceuta antes de empezar a consumir suplementos. Hay riesgos para personas que toman altos niveles de calcio. $\mathrm{Su}$ doctor o farmaceuta puede también decirle que tipo de calcio es el correcto para su consumo.

Para mejor absorción, tómese estos suplementos con las comidas. Es también buena idea consumir el calcio de las comidas o suplementos a través del día y no todo de una. El cuerpo solo puede absorber $500 \mathrm{MG}$ de calcio por vez.

Para ser absorbidas, las tabletas de calcio deben ser disueltas en el estómago. Es mejor comprar sus suplementos de un fabricante confiable para asegurarle que

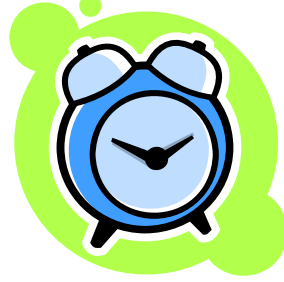
está consiguiendo un suplemento de calidad. Un símbolo USP (United States Pharmacopeia) en la botella significa que el suplemento se disolverá y que no contiene plomo.

Algunos alimentos naturalmente bajos en sodio como lo es el jugo de naranja, están disponibles con calcio adherido. Estos alimentos fortificados son una buena alternativa para personas que se limitan a otros ingresos de calcio.

Las etiquetas nutricionales muestran el contenido de calcio en los alimentos. Dése cuenta que el porcentaje diario en la etiqueta esta basado en 1000 miligramos (vea la Tabla 4).

Tabla 4. Alimentos Suplementados con Calcio

\begin{tabular}{|l|c|c|}
\hline & $\begin{array}{c}\text { \% Valor } \\
\text { Diario }\end{array}$ & $\begin{array}{c}\text { Calcio } \\
\text { (mg) }\end{array}$ \\
\hline $\begin{array}{l}\text { Cereal, fortificado, 1 } \\
\text { taza }\end{array}$ & $10-100 \%$ & $100-1000$ \\
\hline $\begin{array}{l}\text { Jugo de naranja, } \\
\text { fortificado, 6 onzas }\end{array}$ & $26-45 \%$ & $260-450$ \\
\hline $\begin{array}{l}\text { Bebidas de Soya, } \\
\text { fortificadas, 8 onzas }\end{array}$ & $8-50 \%$ & $80-500$ \\
\hline
\end{tabular}

\section{Vitamina D}

La vitamina D promueve la absorción. Esta vitamina se produce en la piel después de haber tenido exposición solar. Esta también adherida a la leche fortificada y algunos otros alimentos fortificados. 
El consumo recomendado de vitamina D son 5 microgramos $(\mu \mathrm{g})(200 \mathrm{IU})$ para aquellas personas entre los 19 y los 50 años de edad, 10 $\mu \mathrm{g}(400 \mathrm{IU})$ para aquellos entre 51 y 70 y $15 \mu \mathrm{g}$

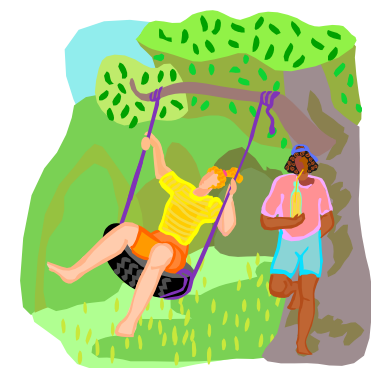

(600 IU) para aquellos mayores de 70. Los niños y los adolescentes deberían consumir $5 \mu \mathrm{g}$ de vitamina $\mathrm{D}$ por día. Pasando de 10 a 15 minutos en el sol cada día (sin bloqueador solar) y alimentándose de comidas fortificadas, le ayudaría a obtener la cantidad suficiente de vitamina D.

Recientes estudios han demostrado que hay personas que podrían tener deficiencia en vitamina D así incrementando el consumo requerido en un futuro. Esto es especialmente verdad para adultos mayores, quienes están menos dispuestos s la exposición solar.

Consumir suficiente calcio y vitamina $\mathrm{D}$ ayuda al cuerpo a construir hueso y disminuir el riesgo de osteoporosis. Alimentarse de una gran variedad de alimentos de todos los grupos en cantidades recomendadas asegurará el consumo de otros minerales que promueven la buena salud de los huesos. Para mayor información acerca de nutrición y recomendaciones para una dieta saludable vaya a http://MyPyramid.gov.

La actividad física, incluyendo ejercicio del peso, es el próximo factor de estilo de vida que promueve la buena salud de nuestros huesos.

\section{Muévalo o Piérdalo}

El ejercicio es importante para prevenir la osteoporosis. La expresión "Muévalo o piérdalo" se puede aplicar a los huesos.

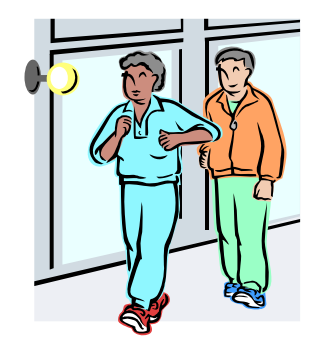

Mientras que el cuerpo se esta moviendo, lo músculos hacen fuerza hacia los huesos. El resultado del estrés en el hueso causa que los minerales (sobretodo el calcio) se depositen en el hueso. Esto hace que el hueso se haga más denso y fuerte.

El mejor tipo de ejercicio para fortalecer los huesos es alzar peso. El ejercicio más recomendado es caminar rápido. Entrenamiento con pesas o maquinas también puede ayudar a fortalecer los huesos.

Mantener un programa de ejercicio en un periodo de largo tiempo podría ser difícil para algunas personas.

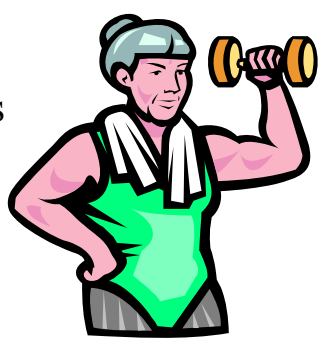

Escoger un ejercicio que disfrute es importante. Salir a caminar con un amigo o caminar en un parque que le guste, por ejemplo, podría ayudar a animar su actividad para tener un estilo de vida activo.

Algunas ideas para incorporar la actividad física a su rutina diaria podrían ser:

- Caminar durante la hora del almuerzo o algún otro tiempo libre en el trabajo.

- Estacionar en la parte de atrás de los parqueaderos cuando vaya trabajar o al centro comercial.

- Use las escaleras en vez de el elevador

- Haga algunas de sus diligencias caminando.

- Camine hasta la oficina de su compañero de trabajo en vez de usar el correo electrónico para comunicarse.

- Relájese con un deporte favorito en vez de relajarte al frente del televisor.

- Cuando vea televisión haga calisténicos o camine por la casa mientras están en propagandas.

Es importante escoger actividades que no incrementen el riego de fractura por una caída $u$ otro accidente. Las personas que no han estado activas en algún tiempo, deben empezar despacio; vea a un doctor antes de empezar un nuevo programa de ejercicio.

\section{Consejo para Adolescentes y Preadolescentes}

La edad es otro factor significativo en la formación del hueso. La masa del hueso se 
incrementa en los periodos de crecimiento, y máximo crecimiento más o menos a los 30 años. Es especialmente importante que mujeres adolescentes y las preadolescentes promuevan un estilo de vida que incluya unos huesos saludables para la perdida de hueso después del periodo menstrual.

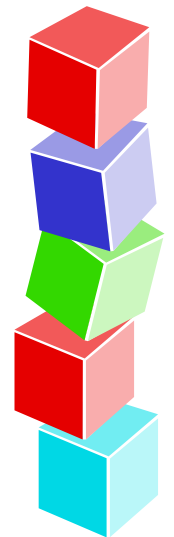

Porque el proceso de contracción del hueso requiere calcio "bloques de contracción," Los adolescentes y preadolescentes deben tener un buen ingreso de calcio. Este grupo está conciente de las dietas y pueden evadir alimentos lácteos creyendo que los va a "engordar." Las niñas adolescentes y preadolescentes deberían ser aconsejadas de usar una variedad de productos bajos o sin grasa con calcio y vitamina $\mathrm{D}$ adherida. Alimentarse de una variedad de frutas y verduras podría darles una variedad de minerales que necesitan, como el magnesio que también colabora con la salud de nuestros huesos.

Los adolescentes y los preadolescentes deberían también tener oportunidades de ejercicio vigoroso cada día, bien sea en la escuela o después de la escuela y en actividades los fines de semana. El levantamiento de peso ayudaría para crear unos huesos fuertes y saludables.

\section{Terapia de Estrógeno}

Durante los años de infancia, una mujer produce una hormona llamada estrógeno. Al llegar a la época de la menopausia, la producción se cae. Esta caída de producción de estrógeno podría contribuir a la perdida de hueso. La Terapia de Reemplazo de la Hormona o (HRT) provee alivio para algunas mujeres que padecen de síntomas severos de la menopausia.

El estrógeno no es recomendado para todas las mujeres, ya que trae muchos efectos secundarios que incluyen riesgo incrementado de infarto y coágulos de sangre. El HRT también podría incrementar el riego de cáncer endometrial. El estrógeno combinado con la progestina disminuye el riesgo de cáncer endometrial, pero cuando las dos hormonas se usan juntas el ciclo menstrual se resume.

La terapia de estrógeno y progestina causa otros efectos secundarios, incluyendo calambre, hinchazón y sensibilidad en el pecho. Los riesgos de la salud incluyen cáncer de ceno, infarto, coágulos de sangre y problemas del corazón.

Si esta considerando la terapia de estrógeno o la de progestina/estrógeno, es muy importante que antes lo consulte con su medico.

\section{Debatiendo un Dilema de Dieta}

A medida que vamos aprendiendo mas sobre la osteoporosis, van apareciendo preguntas que necesitan ser respuestas. Los científicos no están de acuerdo con ningún tratamiento para la osteoporosis. La prevención parece ser lo mejor para esta enfermedad.

Una dieta saludable que incluya suficiente calcio, vitamina $\mathrm{D}$, y otros nutrientes, ejercicio regular, limitación de alcohol, y no fumar son prácticas inteligentes para la

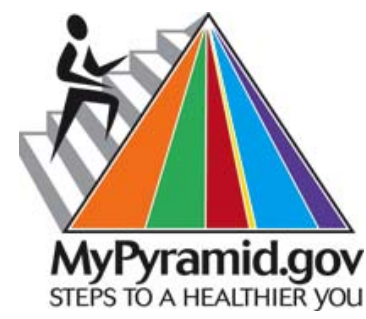
salud en general y pueden ayudar a prevenir la osteoporosis. La terapia de reemplazo de hormona para mujeres en su ciclo de menopausia es todavía un tema controversial, ya que hay un riesgo que podría opacar los beneficios.

Otro aspecto del dilema, es que las comidas lácteas, la fuente principal de calcio en al dieta Americana, no son consumidas por muchos otros grupos étnicos. Esto podría ser por preferencias culturales o por intolerancia a la lactosa. La educación nutricional que es sensible a las diferencias culturales puede ayudar a que las personas hagan selecciones de alimentos que satisfagan sus necesidades nutricionales. 


\section{Resumen}

La osteoporosis es un problema serio y común que tiene muchas causas. Disfrutar de un estilo de vida saludable que incluya comida saludable y actividad física podría ayudar que las personas tengan huesos más saludables durante sus periodos de vida.

\section{Referencias Seleccionadas}

Administration on Aging. Aging Internet Information Notes: Osteoporosis. Available at: http:/www.aoa.gov/prof/notes/notes osteoporosis.asp. Accessed April 26, 2007.

Bupta G, Aronow WS. Hormone replacement therapy - An analysis of efficacy based on evidence. Geriatrics. 57:18-20, 2002.

National Institutes of Health. Dietary Supplement Fact Sheet: Calcium. Available at: http://ods.od.nih.gov/factsheets/calcium.asp. Accessed April 26, 2007.
National Institutes of Health. Menopausal Hormone Therapy Information. Available at: http://www.nih.gov/PHTindex.htm. Accessed April 26, 2007.

National Osteoporosis Foundation. Disease Statistics "Fast Facts." Available at: http://www.nof.org/osteoporosis/diseasefacts. htm. Accessed April 26, 2007.

U.S. Department of Health and Human Services and the Surgeon General's Office. The 2004 Surgeon General's Report on Bone Health and Osteoporosis. Available at:

http://www.hhs.gov/surgeongeneral/library/bone health/content.html. Accessed April 26, 2007.

U.S. Food and Drug Administration. Boning Up on Osteoporosis, 2004. Available at:

http://www.fda.gov/fdac/features/796 bone. html. Accessed April 26, 2007.

Wu F., et al. Prospective 10-year study of the determinants of bone density and bone loss in normal post-menopausal women, including the effect of hormone replacement therapy. Clin Endocrinol, 56:703-11, 2002.

\section{Recetas que contienen Calcio}

\section{Pudín de Arroz a la Antigua ${ }^{a}$}

1 cuarto de leche sin grasa

$1 / 3$ taza de arroz crudo (no lo tape)

$1 / 4$ taza de azúcar

$1 / 2$ cucharadita de sal

$1 / 4$ taza de pasas (Ablándelas en agua caliente si es necesario)

Nueces molidas

Precaliente el horno a 300 grados. Combine la leche, el arroz, el azúcar y la sal en una cacerola con dos cuartos de mantequilla. Cocine sin tapa por 30 minutos. Adhiérale las pasas durante los últimos 30 minutos mientras se cocina y bátalo.

El arroz absorberá la leche. Roséelo con las nueces molidas. Sírvalo caliente o enfríelo con un poco de leche adicional, si así lo desea. Hará 4 porciones.

Cada porción provee aproximadamente:

Calorías: 200

Grasa: $\quad$ Menos de 1 gramo

Calcio: 300 miligramos

a Reimpreso con permiso, National Dairy Board

\section{Frutas Frescas con Salsa de Natillas de Naranja}

$1 \frac{1}{2}$ taza $1 \%$ leche (o úsale sin grasa)

2 cucharadas de azúcar

1 cucharada de maicena

$1 \frac{1}{2}$ cucharadas de cáscaras de naranja

1 yema de huevo 
2 tazas de frutas frescas combinadas en pequeños pedazos (ejemplo. Peras, melocotones, manzanas, uvas, piña, etc.)

En un pequeño sartén combine la leche, el azúcar, la maicena, las cáscaras de naranja y la yema del huevo. Cocínelos y bátalo en medio hasta que hierva. Déjelo hervir por un minuto, bátalo constantemente. Sírvalo caliente o frió sobre las frutas cortadas. Hará dos porciones.

Cada porción proveerá aproximadamente:

Calorías: 270

Grasa: 5 gramos

Calcio: 250 miligramos

\section{Batido para la Mañana}

1 taza $1 \%$ leche (o sin grasa)

1 taza de banano picado congelado

3 cucharadas de concentrado de naranja

congelado

$1 / 2$ cucharaditas de extracto de vainilla puro

Ponga la leche, el banano, la naranja y la vainilla en la licuadora. Cúbrala y lícuela hasta que este suave. Sírvalo de una y esto hará dos porciones.

Cada porción provee aproximadamente:

Calorías: 150

Grasa: $\quad 1.4$ gramos

Calcio: 160 miligramos

\section{Helados de Yogurt (¡Perfectos para niños!)}

1 taza de yogurt natural sin grasa o bajo en grasa 1 banano, picado

1 cucharadita de vainilla

1 taza de jugo de fruta o trozos de frutas

Licue los ingredientes y póngalos en tazas de papel. Congélelos. Póngales una cuchara plástica o un palo de helado en cada taza de yogurt. Para servirlo, ponga la taza al revés y póngale agua caliente hasta que el helado salga. Use las tazas como un sostenedor. Hará de cuatro a cinco porciones de pequeños helados.

$\begin{array}{ll}\text { Calorías: } & 90 \\ \text { Grasa: } & 1 \text { gramo } \\ \text { Calcio: } & 120 \text { miligramos }\end{array}$

\section{Torta Italiana de Arroz ${ }^{\mathrm{b}}$}

$2 / 3$ tazas de arroz crudo (o 2 tazas de arroz cocido)

$1 \frac{1}{3}$ tazas de agua (si esta usando arroz crudo)

1 cebolla, picada

1 cucharada de margarina

16 -onzas lata de pasta de tomate (sin sal) y una lata de agua

$1 / 2$ cucharada de polvo de ajo

1 cucharada de azúcar

Rocíele pimienta

$1 / 2$ cucharada de tomillo entero

$1 / 2$ cucharada de orégano seco, molido

1 cucharada de perejil

$1 \frac{1 / 2}{2}$ taza de queso coteja rallado

$1 / 2$ taza de queso mozarela medio desnatado

Cocine el arroz en agua. En un sartén sofría las cebolla en la margarina. Adhiérale la pasta de tomate, agua, el polvo de ajo, el azúcar, la pimienta, el tomillo, el orégano, y el perejil a la mezcla de la cebolla.

Combine el queso coteja con el arroz.

Ponga $1 / 3$ de la mezcla del arroz en una cacerola que no se pegue. Póngale $1 / 3$ de la salsa de tomate. Continúe alternando las capas, acabando con la salsa de tomate. Después póngale el queso encima. Hornéelo a 325 grados por 30 minutos, hasta que este caliente y burbujeante. Hará seis porciones.

Cada porción provee aproximadamente:

$\begin{array}{ll}\text { Calorías: } & 200 \\ \text { Grasa: } & 5 \text { gramos } \\ \text { Calcio: } & 130 \text { miligramos }\end{array}$

\footnotetext{
${ }^{\mathrm{b}}$ Reprinted with permission from Cooking ala Heart by Linda Hachfeld, MPH, RD and B. Eykyn, MS: (C 2006. Published by Appletree Press, available by calling (800) $322-5679$ or visiting the website, www.appletreepress.com
} 


\section{Cacerola de Coliflor con Maníb}

Por el queso y el maní, esto es mas una entrada vegetariana que un plato adicional. Sírvalo con una ensalada, pan francés, y un postre de frutas. Para mejor variedad, sustituya el brócoli y la col por la coliflor.

1 media cabeza de coliflor, partido en pedazos

1 taza de yogurt natural

1 taza de queso chedar rallado

1 cucharada de harina

2 cucharadas de gránulos con sabor a pollo bajos en sodio

1 cucharada de mostaza seca

$1 / 3$ taza de maní picado

$1 / 3$ taza de migas de pan seco

1 cucharada de margarina

1 cucharada de mejorada seca, molida

En un sartén de tamaño mediano, hierva agua. Adhiérale la coliflor, reduzca el fuego, y déjelo por 15 minutos. Cuélelo.

Mezcle el yogurt, el queso, la harina, los gránulos, y la mostaza. Ponga la coliflor en un plato de hornear de $10 \times 6$ pulgadas o 8 pulgadas y póngale la mezcla del yogurt encima de la coliflor.

Mezcle el maní, las migas de pan, la margarina y la mejorana. Roséelo sobre la coliflor y la salsa. Hornéelo a 400 grados por lo menos por 20 minutos o hasta que ya este burbujeante. Hará cuatro porciones de entradas.

Cada porción proveerá aproximadamente: Calorías: 240

Grasa: 12 gramos

Calcio: $\quad 300$ miligramos

\footnotetext{
${ }^{\mathrm{b}}$ Reprinted with permission from Cooking ala Heart by Linda Hachfeld, MPH, RD and B. Eykyn, MS: (C) 2006. Published by Appletree Press, available by calling (800) 322-5679 or visiting the website, www.appletreepress.com
}

\section{Quiche de Brócoli}

1 libra de brócoli fresco, córtelos en pedazos (o use brócoli congelado)

2 huevos, batidos

$1 / 4$ taza de harina sin blanquear

2 tazas de queso coteja bajo en grasa (1\%)

2 onzas de queso mazarela medio desnatado, rallado

$1 / 4$ taza de perejil fresco, bien picado

1 cucharada de jugo de limón (fresco es mejor)

$1 / 2$ cucharada de albaca seca

$1 / 4$ cucharada de orégano seco

$1 / 4$ cucharada de sal (opcional)

$1 / 4$ cucharada de pimienta

Precaliente el horno a 350 grados. Corte el brócoli en pedazos; pele y corte los vástagos. En un sartén u olla, cocine el brócoli en una pequeña cantidad de agua a fuego medio hasta que el brócoli este crujiente séquelo y píquelo.

Bata los huevos en un recipiente. Adhiérales la harina y mézclelos hasta que estén bien batidos. Adhiérale los ingredientes restantes, mézclelos bien. Rosee un sartén de 9 x 9-pulgadas con aceite vegetal y ponga la mezcla del brócoli. Cocínelos de 35 a 40 minutos a 350 grados. Déjelo enfriar por 2 o 3 minutos. Pártalo en cuadros. Hará cuatro porciones.

Cada porción proveerá aproximadamente:

Calorías: 250

Grasa: 7 gramos

Calcio: 250 miligramos

\section{Raita de Tomate}

1 pepino mediano, pelado

$1 / 2$ cucharada de sal (opcional)

2 tomates medianos sin el corazón, cortados en cubos de $1 / 2$ pulgadas y colados

1 cucharada de cebolla, finamente cortada

1 taza de yogurt natural bajo en grasa

$1 / 4$ taza de perejil fresco, picado

2 cucharadas de cilantro fresco, picado

1 cucharada de comino (al gusto) 
Parta el pepino a la mitad y sáquele las semillas. Pártalo en rodajas delgadas y póngalas en un recipiente. Póngales la sal. Déjelo ahí por 40 minutos. Lávelos y séquelos, presionando el pepino para secarlos bien. Adhiérale los tomates y la cebolla. En un recipiente combine el yogurt, el perejil, el cilantro y el comino; mézclelos bien. Póngalos sobre los vegetales, mézclelos bien. Déjelos enfriar hasta la hora de servirlos. Hará cuatro porciones.

Cada porción provee aproximadamente:

Calorías: 60

Grasa: 1 gramo

Calcio: 130 miligramos

\section{Mouse de Banano}

2 cucharadas de yogurt bajo en grasa (1\%)

4 cucharadas de azúcar

1 cucharada de vainilla

1 banano medio, partido en cuartos

1 taza de yogurt natural sin grasa

8 1/4-pulgadas de pedazos de banano

Ponga la leche, el azúcar, la vainilla y el banano en la licuadora y procéselos por 15 segundos a alta velocidad hasta que se vea blando. Ponga la mezcla en un recipiente pequeño; doble en yogurt. Déjelo ahí. Póngalo sobre los cuatro postres, póngale dos trozos de banano a cada uno antes de servirlo, hará cuatro porciones.

Cada porción provee aproximadamente:

Calorías: 95

Grasa: 1 gramo

Calcio: $\quad 125$ miligramos

\section{Papas Cremosas}

1 libra de papas (las de piel roja están bien)

1 taza de leche sin grasa evaporada

2 cucharadas de margarina baja en grasa

2 cucharada de cebolletas bien picada (o secas)

$1 / 2$ cucharadita de sal

$1 / 4$ cucharada de pimiento Blanca (al gusto)

3 cucharadas de agua

Lave y pele las papas; córtelas en pequeños

trozos. Cocine las papas por 20 o 30 minutos o hasta que les entre fácilmente el tenedor. Mezcle los ingredientes restantes en un recipiente grande. Cuele las papas y póngales la mezcla de la leche. Hará seis porciones.

Cada porción provee aproximadamente:

Calorías: 100

Grasa: 1 gramo

Calcio: 125 miligramos 